\title{
Effects of furan derivatives on biohydrogen fermentation from wet steam-exploded cornstalk and its microbial community
}

Zhidan Liu ${ }^{1}$, Chong Zhang, ${ }^{2, *}$, Linjun Wang ${ }^{2}$, Jianwei $\mathrm{He}^{2}$, Baoming $\mathrm{Li}^{1}$, Yuanhui Zhang ${ }^{1,3}$, Xin-Hui Xing ${ }^{2, *}$

${ }^{1}$ Laboratory of Environment-Enhancing Energy, and Key Laboratory of Agricultural Engineering in Structure and

Environment, Ministry of Agriculture, College of Water Resources and Civil Engineering, China Agricultural

University, Beijing 100083, China;

${ }^{2}$ Key Laboratory for Industrial Biocatalysis, Ministry of Education of China, Department of Chemical

Engineering, Tsinghua University, Beijing 100084, China;

${ }^{3}$ Department of Agricultural and Biological Engineering, University of Illinois at Urbana-Champaign, Urbana, IL 61801, U.S.A.

Corresponding to:

Email: xhxing@tsinghua.edu.cn

Email: chongzhang@tsinghua.edu.cn 


\section{Abstract}

Understanding the role of furan derivatives, furfural (FUR) and 5-hydroxymethyl furfural (HMF), is important for biofuel production from lignocellulosic biomass. In this study, the effects of furan derivatives on hydrogen fermentation from wet steam-exploded cornstalk were investigated. The control experiments with only seed sludge indicated that HMF addition of up to $1 \mathrm{~g} / \mathrm{L}$ stimulated hydrogen production. Similar results were obtained using steam-exploded cornstalk as the feedstock. Hydrogen productivity was increased by up to $40 \%$ with the addition of HMF. In addition, over $90 \%$ of furan derivatives with an initial concentration below $1 \mathrm{~g} / \mathrm{L}$ were degraded. Pyosequencing showed that the addition of HMF and FUR resulted in different microbial communities. HMF led to a higher proportion of the genera Clostridium and Ruminococcaceae, supporting the increased hydrogen production. This study suggested that hydrogen fermentation could be a detoxifying step for steam-exploded cornstalk, and HMF and FUR exhibited different functions for hydrogen fermentation.

\section{Keywords}

Anaerobic fermentation, Biohydrogen, Furan derivatives, Steam-exploded cornstalk 


\section{Introduction}

Waste, otherwise known as low-grade biomass has become a worldwide problem in terms of environment and natural resources. China is one country that produces large amounts of low-grade biomass from agriculture each year. Low-grade biomass mainly consists of plant residues and livestock manure, which has caused serious environmental pollution and social problems, such as the emission of greenhouse gases, non-point source pollution, water eutrophication and pathogen contamination (Chen et al., 2009; Zhang et al., 2010). Waste biomass can be used to generate biohythane i.e. the mixture of biohydrogen and biomethane by two-stage anaerobic fermentation, which offers more environmental and social benefits than fossil-based hythane (Liu et al., 2013; Lu et al., 2009). Compared to biomethane fermentation, two-stage biohythane fermentation maximizes the total energy recovery from biomass and shortens the fermentation time (Liu et al., 2013). Efficient biohydrogen fermentation is therefore the first important step towards harvesting biohythane from low-grade biomass.

Steam explosion has already been recognized as an efficient method to destroy the structure of lignocelluloses, for instance cornstalk, using a high-pressure steam (Hendriks and Zeeman, 2009). However, furan derivatives are released during steam explosion, which are regarded as a notorious fermentation inhibitor ( $\mathrm{Li}$ and Chen, 2008; Wang et al., 2013). The furan derivatives mainly include furfural (FUR) and hydroxymethyl furfural (HMF), which exert negative influence on microbial fermentation through reducing cell growth rate, lowering cell membrane permeability, and inducing reactive oxygen species (Allen et al., 2010; Eva and Barbel, 2000; Koopman et al., 2010). Most studies focused on the influence of furan derivatives on ethanol fermentation (Almeida et al., 2009; Ask et al., 2013; Taherzadeh et al., 1999). Recently, the inhibitory effects of furan derivatives on biohydrogen production have received increasing attention (Cao et al., 2010; Kongjan et al., 2010; Park et al., 2011; Quéméneur et al., 2012; Veeravalli et al., 2013). Specifically, Cao et al. investigated $3 / 27$ 
the effect of fermentation inhibitors, including furan derivatives, on thermophilic hydrogen production from acid pretreated cornstalk by using a pure culture, Thermoanaerobacterium thermosaccharolyticum W16 (Cao et al., 2010). Veeravalli et al. (2013) studied the effects of HMF/FUR (0-1 g/L) on hydrogen production from a glucose-containing medium and found that furans affected the hydrogen production and microbial diversity. Another study (Monlau et al., 2013) reported the inhibition of sunflower stalk hydrolyzates on hydrogen production from a glucose-containing medium. The hydrolyzates contained soluble sugars, and fermentation inhibitors, including furan derivatives and phenolic compounds. The hydrogen productivity was decreased largely to zero with the increase of the hydrolyzates concentration. The dominant population was shifted from Clostridium sp. to Sporolactobacillus sp. It has been suggested that the concentration of the inhibitors should be largely reduced to avoid their negative influence on the microbial metabolism.

As furan derivatives are always regarded as fermentation inhibitors, many studies have been devoted to detoxifying furan derivatives or screening microorganisms tolerant to furan derivatives ( $\mathrm{Li}$ and Chen, 2008; Taherzadeh et al., 1999; Wang et al., 2013; Zhang et al., 2013). Detoxification through biochemical conversion has been employed since the 1960s (Kakinuma and Yamatodani, 1964; Wierckx et al., 2011). The commonly isolated furan-degrading microorganisms are aerobic gram-negative bacteria such as Pseudomonas sp. (Koenig and Andreesen, 1989; Lopez et al., 2004), and Stenotrophomonas sp. (Lopez et al., 2004). Koopman recently isolated a strain, Cupriavidus basilensis HMF 14, capable of selectively degrading HMF and FUR, while leaving sugars intact (Koopman et al., 2010; Wierckx et al., 2010). However, anaerobic degradation of furan derivatives has seldom been reported. Desulfovibrio sp. is the only isolated anaerobic microorganism capable of converting FUR into acetic acid (Boopathy and Daniels, 1991; Brune et al., 1983).

Simultaneous saccharification and hydrogen generation from solid lignocellulosic biomass, such as cornstalk can be realized through anaerobic fermentation (Cheng and Liu, 2011; Lu et al., 2009; Xu et al., 2010). Steam explosion is useful for biomass pretreatment in order to improve the fermentation efficiency of cornstalk for hydrogen $4 / 27$ 
production, which always results in the formation of HMF and FUR as discussed above. Nevertheless, the performance of HMF and FUR in hydrogen fermentation from steam-exploded cornstalk by anaerobic microbial consortium was never reported, which will be presumably totally different from those reported for the fermentation from a glucose-containing medium. This is mainly because hydrogen fermentation from cornstalk is much different from other easily degradable substrates, such as organic wastewater, starch or kitchen wastes.

The purposes of the current study were 1) to compare the effects of HMF and FUR on hydrogen fermentation from wet steam-exploded cornstalk using anaerobic sludge as inocula; 2) to analyze whether furan derivatives can be detoxified through anaerobic hydrogen fermentation; and 3) to elucidate the different impacts of HMF and FUR on microbial communities during hydrogen fermentation from steam-exploded cornstalk using the 454 pyrosequencing technology.

\section{Materials and methods}

\subsection{Inoculum, feedstock and medium}

Seed sludge was collected from an anaerobic digester at the Xiaohongmen Wastewater Treatment Plant (Beijing, China). Heat treatment was accomplished by boiling the samples using $100^{\circ} \mathrm{C}$ hot water for $30 \mathrm{~min} .7 \%(\mathrm{w} / \mathrm{v})$ of the boiled seed sludge was inoculated for hydrogen fermentation. Steam explosion of the cornstalks was carried out with high-pressure steam at $1.5 \mathrm{MPa}, 200{ }^{\circ} \mathrm{C}$ for $5 \mathrm{~min}$ in a steam-explosion reactor (Laihe Company, China). The wet steam-exploded cornstalk had a $28-30 \%$ total solid content (TS) and 73\% VS (volatile solid) of TS. The VS consisted of $70 \%$ of cellulose, $6 \%$ of hemi-cellulose and $24 \%$ of lignin. The raw wet steam-exploded cornstalk contained HMF and FUR at the concentrations of 150 200 $\mathrm{mg} / \mathrm{L}$ and 160 220 mg/L, respectively. The medium included the following (per L): yeast extract, $2.0 \mathrm{~g}$; $\left(\mathrm{NH}_{4}\right)_{2} \mathrm{SO}_{4}, 1.3 \mathrm{~g} ; \mathrm{KH}_{2} \mathrm{PO}_{4}, 1.5 \mathrm{~g}$; $\mathrm{K}_{2} \mathrm{HPO}_{4} \cdot 3 \mathrm{H}_{2} \mathrm{O}, 2.9 \mathrm{~g} ; \mathrm{CaCl}_{2}$, $0.075 \mathrm{~g} ; \mathrm{MgCl}_{2} \cdot 6 \mathrm{H}_{2} \mathrm{O}, 0.2 \mathrm{~g}$; and $\mathrm{FeSO}_{4} \cdot 7 \mathrm{H}_{2} \mathrm{O}, 1.25 \mathrm{mg}$. The wet SEC was diluted with a prepared medium to reach a final TS concentration of $8 \%$ and then it was used 
as the substrate for hydrogen fermentation without any other treatment. The initial $\mathrm{pH}$ of the substrate was adjusted to 6.5 for hydrogen fermentation.

\subsection{Experimental setup and procedure}

The experiment device was a normal-pressure batch bioreactor system, consisting of a 250-mL glass flask (150-ml working volume), gas-tight plastic tubes, sampling valve, and gas balloon. The glass flask served as the anaerobic bioreactor, the produced gas was measured through the gas sampling valve and collected by using the gas balloon, and the fermented broth was evaluated by the sampling port embedded in the flask.

HMF and FUR were employed as model furan derivatives of the cornstalk hydrolysate through steam-explosion. The effects of furan derivatives on hydrogen fermentation from steam-exploded cornstalk were conducted in the following procedure: A control experiment was designed by investigating hydrogen production fed with inoculated seed sludge and externally added furan derivatives in different concentrations $(0 \mathrm{mg} / \mathrm{L}, 10 \mathrm{mg} / \mathrm{L}, 100 \mathrm{mg} / \mathrm{L}, 500 \mathrm{mg} / \mathrm{L}$ and $1000 \mathrm{mg} / \mathrm{L})$ without any cornstalk. The purpose of this experiment was to obtain the baseline of hydrogen production from seed sludge and to study the effects of furan derivatives on the activities of seed sludge. Furan derivatives in five concentrations $(0 \mathrm{mg} / \mathrm{L}, 100 \mathrm{mg} / \mathrm{L}$, $500 \mathrm{mg} / \mathrm{L}, 1000 \mathrm{mg} / \mathrm{L}$ and $2000 \mathrm{mg} / \mathrm{L}$ ) were then applied to hydrogen fermentation from steam-exploded cornstalk using the same seed sludge to study how furan derivatives affected hydrogen fermentation. The microbial communities formed in the hydrogen fermentation with the additions of HMF and FUR were finally analyzed by pyrosequencing. Hydrogen fermentation was performed using closed flasks in a 100 rpm biochemical shaker at $37^{\circ} \mathrm{C}$.

In the verification experiment of hydrogen fermentation capable of utilizing HMF and FUR contained in SEC, a 5-L stirred batch reactor fed with steam-exploded cornstalk (TS, 8\%) was used. The inocula were the same sludge as that used in the flask batch culture. Hydrogen fermentation in the 5-L batch reactor was carried out with an agitating speed of $100 \mathrm{rpm}$, a temperature of $37^{\circ} \mathrm{C}$ and $\mathrm{pH}$ controlled at 6.5. 


\subsection{Analytical methods}

Gas composition was detected as previously described (Liu et al., 2012) using a GC equipped with a thermal conductivity detector and a stainless-steel column packed with TDX-01 (GC112A, China). The detected gases included hydrogen, oxygen, and methane.

Furan derivatives and metabolic intermediates formed during fermentation were analyzed by HPLC (Waters 1525 HPLC) equipped with a refractive index detector (RID) and an Aminex HPX-87H ion exclusion column. $\mathrm{H}_{2} \mathrm{SO}_{4}(5 \mathrm{mmol} / \mathrm{L})$ was used as the mobile phase at a flow rate of $0.6 \mathrm{ml} / \mathrm{min}$. The samples were centrifuged (12000 rpm, $10 \mathrm{~min}$ ) and the supernatant was filtered using a $0.22-\mu \mathrm{m}$ membrane filter before use. The detected intermediates included volatile fatty acids (VFAs) and ethanol.

The phylogenetic diversity was analyzed using a 454 pyrosequencing technique in order to study the influence of additional furan derivatives on microbial communities of hydrogen fermentation. The samples were obtained from the batch reactor induced by furan derivatives after the experiments. The initial concentration of added HMF or FUR was $500 \mathrm{mg} / \mathrm{L}$. The microbial cells were washed using a phosphate buffer $(\mathrm{pH}=$ 7) and centrifuged to isolate the DNA. The genomic DNA was extracted using an E.Z.N.A DNA Isolation Kit (OMEGA Biotec., USA), according to the manufacturer's instructions. The bacterial 16S rRNA gene fragments were PCR-amplified using primers 8F (5'-AGAGTTTGATCCTGGCTCAG-3') and 533R (5'-TTACCGCGGCTGCTGGCAC-3'). Each 20- $\mu$ L PCR reaction mixture included 4 $\mu \mathrm{L}$ of $5 \times$ FastPfu buffer, $2 \mu \mathrm{L}$ of $2.5 \mathrm{mM}$ dNTPs, $0.4 \mu \mathrm{L}$ of forward primer $(5 \mu \mathrm{M})$, $0.4 \mu \mathrm{L}$ of reverse primer $(5 \mu \mathrm{M}), 0.5 \mu \mathrm{L}$ of DNA template, $0.4 \mu \mathrm{L}$ of Fastfu polymerase, and added $\mathrm{ddH}_{2} \mathrm{O}$ to make up to a final volume of $20 \mu \mathrm{L}$. The PCR temperatures were as follows: $95^{\circ} \mathrm{C}$ for $2 \mathrm{~min} ; 25$ cycles consisting of $95^{\circ} \mathrm{C}$ for $30 \mathrm{~s}$, $55^{\circ} \mathrm{C}$ for $30 \mathrm{~s}, 72^{\circ} \mathrm{C}$ for $30 \mathrm{~s}$, and $72^{\circ} \mathrm{C}$ for $5 \mathrm{~min}$. Duplicate PCR products were pooled and purified using an AXYGEN gel extraction kit (Axygen, USA). The purified PCR products were used for pyrosequencing. Pyrosequencing was carried out using a 454 Life Sciences Genome Sequencer FLX Titanium instrument (Roche) $7 / 27$ 
(Shanghai Majorbio Bio-Pharm Biotechnology Co., Ltd., Shanghai, China).

The sequences were analyzed using a Quantitative Insights into Microbial Ecology (QIIME) platform. First, the multiplexed reads were assigned to samples based on their nucleotide barcodes. In addition, during this step, these sequences were screened using the default parameters: the minimum quality score $=25$ and the minimum/maximum length $=200 / 1000$. Furthermore, no ambiguous bases were allowed, and no mismatches were allowed in the primer sequence. Then, all the sequences from all the samples were clustered into operational taxonomic units (OTUs) by UCLUST. Once clustered, a representative sequence from each OTU was picked for downstream analysis, and taxonomic identity was assigned to each representative sequence using BLAST with a 0.001 maximum e-value.

\section{Results and discussion}

\subsection{Effects of furan derivatives on hydrogen production from seed sludge control}

To obtain the baseline of hydrogen production from seed sludge and study the effects of furan derivatives on the activities of seed sludge, a control experiment was first designed by investigating hydrogen fermentation fed with inoculated seed sludge and furan derivatives without cornstalk. Table 1 shows the influence of furan derivatives on the hydrogen production from only the inoculated seed sludge. With the increase of HMF concentration from 0 to $1000 \mathrm{mg} / \mathrm{L}$, hydrogen production was increased, reduced, and again increased, indicating the effect of HMF on dark fermentation. Compared with HMF, FUR more strongly interfered with hydrogen production (Table 1). Hydrogen was not generated at any concentration of FUR. These results revealed that HMF and FUR might undergo different mechanisms, which affect the anaerobic fermentation. Only the functions of furan derivatives were discussed in the previous study (Ask et al., 2013; Eva and Barbel, 2000) and whether HMF and FUR had different effects on the anaerobic fermentation was unknown. As reported by Almeida (2009), the inhibitory effect of FUR has been known for over 30 years, whereas the 
mechanism of HMF's effect on the functions of anaerobic bacteria is still not fully understood. The different effects of HMF and FUR found here might provide further evidences for their different mechanisms on hydrogen fermentation. The analysis of the fermentation broth through HPLC (data not shown) showed that the concentration of the released VFAs was associated with the increase of the HMF concentration. The produced hydrogen might be because HMF or HMF affected the activity of seed sludge, and the mechanism needs to be further studied. However, one straightforward fact revealed by these experiments was that HMF up to a certain concentration could improve the hydrogen fermentation.

\subsection{Effects of furan derivatives on hydrogen production from steam-exploded cornstalk}

Raw wet steam-exploded cornstalk contained HMF and FUR with concentrations of 150 200 mg/L and 160 220 mg/L, respectively. The additional HMF and FUR in different concentrations were employed as model chemicals to study the effect of furan derivatives on hydrogen fermentation from steam-exploded cornstalk. Figure 1 shows that the external addition of furan derivatives significantly affected the hydrogen production from steam-exploded cornstalk in a 0.25 -L normal-pressure flask batch culture using anaerobic sludge as inocula. Similar to the inoculated seed sludge experiment (Table 1), hydrogen production was first increased and then reduced with the increase of the HMF concentration. The highest volumetric hydrogen production rate was around $0.4 \mathrm{~L} / \mathrm{L}$ at a $\mathrm{HMF}$ concentration of $100 \mathrm{mg} / \mathrm{L}$, which was $40 \%$ higher than that observed in the control without the addition of HMF. HPLC analysis showed significant HMF degradation during the hydrogen fermentation. Over $90 \%$ of HMF was degraded after hydrogen fermentation when the HMF concentration was below $1000 \mathrm{mg} / \mathrm{L}$ (Fig. 1b). The degradation rate of HMF was decreased to $36 \%$ at $2000 \mathrm{mg} / \mathrm{L}$ of HMF. The HPLC results (Table 2) show that the total concentration of VFAs was increased with the increase of the HMF concentration. 
Unlike HMF, FUR clearly inhibited hydrogen fermentation (Fig. 1c). Furthermore, the total concentration of VFAs did not clearly increase with the increase of the FUR concentration. However, a similar degradation rate of FUR was found during hydrogen fermentation. The furan derivatives in the control experiment without sludge inoculation exhibited no degradation during the same period, suggesting that the degradation of furan derivatives resulted from anaerobic conversion. The combined addition of HMF and FUR (Fig. 1e) negatively impacted hydrogen production. Surprisingly, the inhibitory strength of the combined addition of HMF and FUR was weaker than that of only FUR at the same concentration. This finding was different from the results obtained in previous studies on ethanol fermentation (Eva and Barbel, 2000; Pienkos and Zhang, 2009), in which the combination of inhibitors always produced synergistic or interaction inhibitory effects. Note that the data presented in Fig.1a, Fig.1c and Fig.1e were the average of three repeated experiments. The standard deviations of hydrogen productivity $(\mathrm{L} / \mathrm{L})$ with the additions of HMF and/or FUR were similar in a range of $0 \sim 0.2 \mathrm{~L} / \mathrm{L}$ (data not shown). To date, there has only been a few studies focusing on the influence of inhibitors on hydrogen fermentation by the pure culture, which was also accompanied with the synergistic inhibitory effect (Cao et al., 2010). In the present study, HMF was found to be used as a kind of regulator capable of affecting the anaerobic metabolism for hydrogen fermentation using anaerobic sludge as inocula (Table 2 and Fig.1).

Another interesting finding was that the production of VFAs during hydrogen fermentation highly depended on the types of furan derivatives present (Table 2). HMF, FUR, and FMF+FUR at various concentrations led to different distributions of VFAs in the fermented broth. Under all the conditions, acetic acid was the dominant VFA and butyric acid was largely reduced at $2000 \mathrm{mg} / \mathrm{L}$. The production of lactic acid and formic acid was enhanced or stimulated during hydrogen fermentation with the external addition of HMF or HMF+FUR, suggesting that lactate fermentation might take place at higher concentrations of HMF. In comparison, the production of succinic acid was stimulated with the addition of FUR, whereas 2, 3-butanediol production was enhanced with the addition of HMF+FUR. Lactic acid was not found and lactate $10 / 27$ 
fermentation was even restrained at higher concentrations of FUR, probably because of its stronger inhibition than HMF. This speculation is supported by other results (Fig.1, Table 1). In addition, the $\mathrm{pH}$ values after hydrogen fermentation (Table 2) mostly decreased with the increase of HMF/FUR concentrations although the initial $\mathrm{pH}$ values for all the batch cultures were identical at 6.5 . The lowest $\mathrm{pH}$ values were 5.65 at $1000 \mathrm{mg} / \mathrm{L}$ (HMF), 5.39 at $2000 \mathrm{mg} / \mathrm{L}$ (FUR), and 5.72 at $1000 \mathrm{mg} / \mathrm{L}$ (HMF+FUR). The different final $\mathrm{pH}$ values might have resulted from the different microbial metabolisms caused by the addition of furan derivatives in different concentrations. These results revealed that HMF and FUR might undergo different mechanisms, which affect anaerobic fermentation of cornstalk. Besides this, a straightforward conclusion was that HMF or HMF+FUR induced a similar distribution of VFAs. Brune (1983) and Boopathy (1991) also found that FUR was converted to acetic acid by anaerobic fermentation using a sulfate-reducing bacterium, Desulfovibrio sp. The effects of furan derivatives on hydrogen fermentation might be associated with the metabolism of VFAs. However, it is difficult to clarify the roles of furan derivatives on the production of VFAs, and a future study will be needed in order to explain their roles.

To examine the changes of furan derivatives during hydrogen fermentation, a 5-L stirred batch reactor, fed with steam-exploded cornstalk in a final concentration of $8 \%$ (TS), was run using anaerobic sludge as inocula under mesophilic conditions with a controlled $\mathrm{pH}$. Only the intrinsic furan derivatives released from the cornstalk after steam explosion were included in the fermentation system. As illustrated in Fig. 2, the initial concentrations of HMF and FUR in the bulk solution were similar at around 50 $\mathrm{mg} / \mathrm{L}$. Both HMF and FUR were degraded within one week during the hydrogen fermentation process. The hydrogen productivity reached over $0.5 \mathrm{~L} / \mathrm{L}$ after four days' operation, higher than the results in the 0.25 -L batch flask culture. The highest hydrogen content was $60 \%$ within the first $24 \mathrm{~h}$. The relatively high hydrogen productivity using 5-L reactor was due to better mass transfer and control of the $\mathrm{pH}$ at 6.5 during the fermentation. From the perspective of gaseous biofuel production, the results shown in Figs. 1 and 2 suggest that hydrogen production might serve as a $11 / 27$ 
simultaneous detoxification process, thus benefiting subsequent biomethane production from VFAs for the purpose of biohythane harvesting from agricultural biomass (Liu et al., 2013).

\subsection{Effects of furan derivatives on microbial communities during hydrogen}

\section{fermentation}

To further elucidate the impact of furan derivatives on hydrogen fermentation, the diversity of microbial communities formed during the hydrogen fermentation with the addition of furan derivatives was examined using 454 pyrosequencing technology. Hydrogen fermentation from wet steam-exploded cornstalk fed with furan derivatives at an initial concentration of $500 \mathrm{mg} / \mathrm{L}$ was chosen for the analysis of the microbial communities. The reason for using $500 \mathrm{mg} / \mathrm{L}$ of furan derivatives was due to the fact that at $500 \mathrm{mg} / \mathrm{L}$, hydrogen fermentation from steam-exploded cornstalk was clearly enhanced by HMF other than FUR (Fig.1). A total of 24,598 and 25,136 high-quality reads were obtained for the microbial communities in hydrogen fermentation with the respective addition of HMF and FUR, with an average length of 478 bp (Table 3). Ace and Chao indexes indicate the richness of the microbial communities, whereas Shannon and Simpson indexes analyze the microbial diversity . At a 3\% distance, the Chao indexes were 2529 (for HMF) and 2604 (for FUR), whereas the Shannon indexes were 5.5 (for HMF) and 5.74 (for FUR). Based on the Chao and Shannon indexes, it was concluded that the microbial communities formed in the fermentation with the addition of HMF and FUR had similar diversities.

Figure 3 shows the rarefaction curve of microbial communities formed in the fermentation with the addition of furan derivatives. Species richness could be calculated through the analysis of obtained OTUs in the rarefaction curve. A total of 1680,1227 , and 756 OTUs were obtained at 3,5 , and $10 \%$ distances for the microbial communities formed with the addition of HMF, while 1775, 1300, and 798 OTUs were obtained for those with the addition of FUR. Fig. 3 indicates that HMF and FUR resulted in microbial communities with similar species richness, supporting the results 
of diversity analysis (Table 3). Another aspect was that even after sampling 20,000 reads, new microbial species could still be detected. The estimated total OTUs through the ExpAssoc model were 2096 (HMF) and 2157 (FUR).

To compare the phylogenetic difference in microbial communities formed in the hydrogen fermentation with the respective additions of furan derivatives, the qualified reads were classified into six different levels, including phylum, class, order, family, genus, and species (Fig. 4). The phylum-level classification of microbial communities is shown in Fig. 4A. The additions of HMF and FUR led to a different distribution of microbial phyla. The dominant phyla were Chloroflexi, Firmicutes, and Proteobacteria in both cases, and the sum of the three phyla accounted for $79.28 \%$ (for HMF) and 75.74\% (for FUR) of the total reads, respectively. HMF addition resulted in a higher distribution of Firmicutes (relative abundance $=39.1 \%$ ) than FUR addition (relative abundance $=16.53 \%$ ). Many hydrogen-producing microorganisms, especially Clostridium sp., are known to belong to the phylum Firmicutes (Im et al., 2012). Thus, the analysis of microbial communities might provide a proof for the effects of HMF and FUR on hydrogen fermentation. The findings shown in Figs. 1 and 2 demonstrate that HMF at certain concentrations stimulated hydrogen production. This was further confirmed by pyrosequencing, which revealed that the HMF addition resulted in an over two-fold higher proportion of Firmicutes than the FUR addition (Fig. 4).

The class-level classification of microbial communities is illustrated in Fig. 4B. The additions of HMF and FUR resulted in two different microbial community structures during hydrogen fermentation. With respect to the microbial class accounting for more than $5 \%$ of the total composition, the microbial community with the addition of HMF consisted of Anaerolineae (21.6\%), Bacilli (7.35\%), Betaproteobacteria (7.34\%), and Clostridia (31.71\%), whereas the FUR addition led to the microbial community consisting of Alphaproteobacteria (8.06\%), Anaerolineae (29.99\%), Betaproteobacteria (9.87\%), Clostridia (15.28\%), and Gammaproteobacteria (5.78\%). Furthermore, higher proportions of Clostridia and Bacilli were found during hydrogen 
fermentation with the addition of HMF, whereas more Proteobacteria were observed during fermentation with the addition of FUR.

The classification with respect to the genus provided a detailed account of the microbial communities formed in the hydrogen fermentation with furan derivatives (Table 4). The biggest population during hydrogen fermentation amended with HMF or FUR was Anaerolineaceae ( $>13 \%$ under both conditions). Anaerolineaceae was reported as the anaerobic genera isolated from anaerobic sludge requiring yeast extract for growth (Yamada, 2006), which was similar to the cultivation conditions of the current study. Compared to the FUR addition, the dominant populations during hydrogen fermentation with the addition of HMF were Clostridium (11.77\%) and Ruminococcaceae $(6.85 \%)$, which are two notable hydrogen-producing genera having common characteristics of being anaerobic, gram-positive, and able to form spores (Quéméneur et al., 2012; Ren et al., 2009). Furthermore, Ruminococcaceae has been mainly isolated from rumen, and it has been observed to typically participate in the hydrolysis of cellulosic biomass (Liu et al., 2012; Pavlostathis et al., 1988). The identification of the genus Ruminococcaceae in the present study supported the function for the degradation of cornstalk. Besides, Lactobacillus (5.75\%) was found among the microbial communities formed in the fermentation by the addition of HMF, suggesting that the production of lactic acid was resulted from the addition of HMF (Table 2).

Another important aspect was the examination of the microorganisms capable of degrading furan derivatives. The majority of the isolated furan-degrading microorganisms have been reported to belong to aerobic gram-negative bacteria (Wierckx et al., 2011). In addition, other bacteria, such as Cupriavidus basilensis HMF14 and Arthrobacter nicotianae, have also been reported to be capable of degrading furanic aldehydes (Wierckx et al., 2010). Arthrobacter sp., belonging to the class Actinobacteria, was also observed in the present study among the microbial communities formed in the fermentation with additions of HMF (1.72\%) and FUR $(3.62 \%)$ addition. Nevertheless, there were only a few studies focusing on the anaerobic degradation of furan derivatives (Brune et al., 1983; Wierckx et al., 2011). 
Desulfovibrio sp. is the only reported anaerobic strain capable of degrading FUR to acetic acid (Boopathy and Daniels, 1991; Brune et al., 1983), which was not found in the present study. In addition, the anaerobic conversion of HMF has been seldom studied. This study demonstrated the efficient degradation of furan derivatives under anaerobic conditions, indicating that further investigation on the degradation mechanisms of furan derivatives by anaerobic fermentation will be necessary.

\section{Conclusions}

The current study demonstrated that two furan derivatives, HMF and FUR, had different influence on hydrogen fermentation from steam-exploded cornstalk. The addition of HMF at less than $1 \mathrm{~g} / \mathrm{L}$ stimulated hydrogen production, while the addition of FUR inhibited hydrogen fermentation. In addition, most furan derivatives were degraded during hydrogen fermentation at an initial concentration below $1 \mathrm{~g} / \mathrm{L}$. Pyosequencing analysis revealed that the addition of HMF and FUR resulted in different microbial communities. HMF stimulated higher proportion of the genera Clostridium and Ruminococcaceae, further supporting the different functions of HMF and FUR for hydrogen fermentation.

\section{Acknowledgments}

This work was supported by the National Key Technology Support Program (2014BAD02B03), NSFC project (21106080), the Project of the National Basic Research Program of China (973 Plan) (2011CB707404), NSFC-JST Joint Project (21161140328), and Chinese Universities Scientific Fund (2012RC030). The authors thank Jamison Watson (University of Illinois at Urbana-Champaign) for helpful suggestions that improved the manuscript.

\section{References}


[1] Allen, S.A., Clark, W., McCaffery, J.M., Cai, Z., Lanctot, A., Slininger, P.J., Liu, Z.L., Gorsich, S.W., 2010. Furfural induces reactive oxygen species accumulation and cellular damage in Saccharomyces cerevisiae. Biotechnol. Biofuels, 3, 2.

[2] Almeida, J.R.M., Bertilsson, M., Gorwa-Grauslund, M.F., Gorsich, S., Lidén, G., 2009. Metabolic effects of furaldehydes and impacts on biotechnological processes. Appl. Microbiol. Biotechnol., $82,625-638$.

[3] Ask, M., Bettiga, M., Mapelli, V., Olsson, L., 2013. The influence of HMF and furfural on redox-balance and energy-state of xylose-utilizing Saccharomyces cerevisiae. Biotechnol. Biofuels, 6.

[4] Boopathy, R., Daniels, L., 1991. Isolation and characterization of a furfural degrading sulfate-reducing bacterium from an anaerobic digester. Curr. Microbiol., 23, 327-332.

[5] Brune, G., Schoberth, S., Sahm, H., 1983. Growth of a strictly anaerobic bacterium on furfural (2-Furaldehyde). Appl. Environ. Microbiol., 46, 1187-1192.

[6] Cao, G.-L., Ren, N.-Q., Wang, A.-J., Guo, W.-Q., Xu, J.-F., Liu, B.-F., 2010. Effect of lignocellulose-derived inhibitors on growth and hydrogen production by Thermoanaerobacterium thermosaccharolyticum W16. Int. J. Hydrogen Energ., 35, 13475-13480.

[7] Chen, L., Xing, L., Han, L., 2009. Renewable energy from agro-residues in China: Solid biofuels and biomass briquetting technology. Renew. Sust. Energ. Rev., 13, 2689-2695.

[8] Cheng, X.Y., Liu, C.Z., 2011. Hydrogen production via thermophilic fermentation of cornstalk by Clostridium thermocellum. Energ. Fuel, 25, 1714-1720.

[9] Eva, P., Barbel, H.H., 2000. Fermentation of lignocellulosic hydrolysates. II inhibitors and mechanisms of inhibition. Bioresour. Technol., 74, 25-33.

[10] Hendriks, A.T.W.M., Zeeman, G., 2009. Pretreatments to enhance the digestibility of lignocellulosic biomass. Bioresour. Technol., 100, 10-18.

[11] Im, W.T., Kim, D.H., Kim, K.H., Kim, M.S., 2012. Bacterial community analyses by pyrosequencing in dark fermentative H2-producing reactor using organic wastes as a feedstock. Int. J. Hydrogen Energ., 37, 8330-8337.

[12] Kakinuma, A., Yamatodani, S., 1964. L-Glutamic acid formation from 2-furoic acid by soil bacteria. Nature, 201, 420-421.

[13] Koenig, K., Andreesen, J., 1989. Molybdenum involvement in aerobic degradation of 2-furoic acid by Pseudomonas putida Fu1. Appl. Environ. Microbiol., 55, 1829-1834.

[14] Kongjan, P., O-Thong, S., Kotay, M., Min, B., Angelidaki, I., 2010. Biohydrogen production from wheat straw hydrolysate by dark fermentation using extreme thermophilic mixed culture. Biotechnol. Bioeng., 105, 899-908.

[15] Koopman, F., Wierckx, N., de Winde, J.H., Ruijssenaars, H.J., 2010. Identification and characterization of the furfural and 5-(hydroxymethyl)furfural degradation pathways of Cupriavidus basilensis HMF14. Proc. Natl. Acad. Sci. U S A, 107, 4919-4924.

[16] Li, H., Chen, H., 2008. Detoxification of steam-exploded corn straw produced by an industrial-scale reactor. Process Biochem., 43, 1447-1451.

[17] Liu, Z., Lv, F., Zheng, H., Zhang, C., Wei, F., Xing, X.-H., 2012. Enhanced hydrogen production in a UASB reactor by retaining microbial consortium onto carbon nanotubes (CNTs). Int. J. Hydrogen Energ., 37, 10619-10626. 
[18] Liu, Z., Zhang, C., Lu, Y., Wu, X., Wang, L., Wang, L., Han, B., Xing, X.-H., 2013. States and challenges for high-value biohythane production from waste biomass by dark fermentation technology. Bioresour. Technol., 135, 292-303.

[19] Lopez, M.J., Nichols, N.N., Dien, B.S., Moreno, J., Bothast, R.J., 2004. Isolation of microorganisms for biological detoxification of lignocellulosic hydrolysates. Appl. Microbiol. Biotechnol., 64, 125-131.

[20] Lu, Y., Lai, Q., Zhang, C., Zhao, H., Ma, K., Zhao, X., Chen, H., Liu, D., Xing, X.-H., 2009. Characteristics of hydrogen and methane production from cornstalks by an augmented two- or three-stage anaerobic fermentation process. Bioresour. Technol., 100, 2889-2895.

[21] Monlau, F., Aemig, Q., Trably, E., Hamelin, J., Steyer, J.-P., Carrere, H., 2013. Specific inhibition of biohydrogen-producing Clostridium sp. after dilute-acid pretreatment of sunflower stalks. Int. J. Hydrogen Energ., 38, 12273-12282.

[22] Park, J.-H., Yoon, J.-J., Park, H.-D., Kim, Y.J., Lim, D.J., Kim, S.-H., 2011. Feasibility of biohydrogen production from Gelidium amansii. Int. J. Hydrogen Energ., 36, 13997-14003.

[23] Pavlostathis, S., Miller, T., Wolin, M., 1988. Fermentation of Insoluble cellulose by continuous cultures of Ruminococcus albus. Appl. Environ. Microbiol., 54, 2655-2659.

[24] Pienkos, P.T., Zhang, M., 2009. Role of pretreatment and conditioning processes on toxicity of lignocellulosic biomass hydrolysates. Cellulose, 16, 743-762.

[25] Quéméneur, M., Hamelin, J., Barakat, A., Steyer, J.-P., Carrère, H., Trably, E., 2012. Inhibition of fermentative hydrogen production by lignocellulose-derived compounds in mixed cultures. Int. J. Hydrogen Energ., 37, 3150-3159.

[26] Ren, N., Wang, A., Cao, G., Xu, J., Gao, L., 2009. Bioconversion of lignocellulosic biomass to hydrogen: Potential and challenges. Biotechnol. Adv., 27, 1051-1060.

[27] Taherzadeh, M.J., Gustafsson, L., Niklasson, C., Lidén, G., 1999. Conversion of furfural in aerobic and anaerobic batch fermentation of glucose by Saccharomyces cerevisiae. J. Biosci. Bioeng., 87, 169-174.

[28] Veeravalli, S.S., Chaganti, S.R., Lalman, J.A., Heath, D.D., 2013. Effect of furans and linoleic acid on hydrogen production. Int. J. Hydrogen Energ., 38, 12283-12293.

[29] Wang, X., Yomano, L.P., Lee, J.Y., York, S.W., Zheng, H., Mullinnix, M.T., Shanmugam, K.T., Ingram, L.O., 2013. Engineering furfural tolerance in Escherichia coli improves the fermentation of lignocellulosic sugars into renewable chemicals. Proc. Natl. Acad. Sci. U S A, 110, 4021-4026.

[30] Wierckx, N., Koopman, F., Bandounas, L., de Winde, J.H., Ruijssenaars, H.J., 2010. Isolation and characterization of Cupriavidus basilensis HMF14 for biological removal of inhibitors from lignocellulosic hydrolysate. Microb. Biotechnol., 3, 336-343.

[31] Wierckx, N., Koopman, F., Ruijssenaars, H.J., Winde, J.H., 2011. Microbial degradation of furanic compounds: biochemistry, genetics, and impact. Appl. Microbiol. Biotechnol., 92, 1095-1105.

[32] Xu, J., Ren, N., Su, D., Qiu, J., 2010. Bio-hydrogen production from acetic acid steam-exploded corn straws by simultaneous saccharification and fermentation with Ethanoligenens harbinense B49. Int. J. Energ. Res., 34, 381-386.

[33] Yamada, T., 2006. Anaerolinea thermolimosa sp. nov., Levilinea saccharolytica gen. nov., sp. nov. and Leptolinea tardivitalis gen. nov., sp. nov., novel filamentous anaerobes, and description of the new classes Anaerolineae classis nov. and Caldilineae classis nov. in the bacterial phylum Chloroflexi. Int. J. Syst. Evol. Microbiol., 56, 1331-1340. 
[34] Zhang, D., Ong, Y.L., Li, Z., Wu, J.C., 2013. Biological detoxification of furfural and 5-hydroxyl methyl furfural in hydrolysate of oil palm empty fruit bunch by Enterobacter sp. FDS8. Biochem. Eng. J., 72, 77-82.

[35] Zhang, J., auzerall, D.L.M., Zhu, T., Liang, S., Ezzati, M., Remais, J.V., 2010. Environmental health in China: progress towards clean air and safe water. Lancet, 375, 1110-1119. 
Table 1 Effects of furan derivatives on hydrogen production from the inoculated seed sludge at the respective addition of HMF and FUR $(n=3)$. The concentrations of furan derivatives were $0,10,100,500$ and $1000 \mathrm{mg} / \mathrm{L}$.

\begin{tabular}{|c|c|c|c|c|c|}
\hline \multirow[t]{2}{*}{$\begin{array}{l}\text { Furan } \\
\text { derivatives }\end{array}$} & \multicolumn{5}{|c|}{$\begin{array}{l}\text { Hydrogen productivity }(\mathrm{mL} / \mathrm{L}) \text { with the additions of furan } \\
\text { derivatives in different concentrations }\end{array}$} \\
\hline & $0 \mathrm{mg} / \mathrm{L}$ & $10 \mathrm{mg} / \mathrm{L}$ & $100 \mathrm{mg} / \mathrm{L}$ & $500 \mathrm{mg} / \mathrm{L}$ & $1000 \mathrm{mg} / \mathrm{L}$ \\
\hline HMF & 0 & $0.37 \pm 0.20$ & $0.02 \pm 0.02$ & $0.13 \pm 0.08$ & $0.58 \pm 0.09$ \\
\hline FUR & 0 & 0 & 0 & 0 & 0 \\
\hline
\end{tabular}


Table 2 Analysis of metabolites $(\mathrm{mM})$ and $\mathrm{pH}$ of biohydrogen production from steam-exploded cornstalk amended with furan derivatives after $66 \mathrm{~h}$ of cultivation $(n=3)$. The concentrations of furan derivatives were $0(A), 100(B), 500$ (C), 1000 (D) and $2000 \mathrm{mg} / \mathrm{L}(\mathrm{E})$.

\begin{tabular}{|c|c|c|c|c|c|c|c|c|c|}
\hline & $\begin{array}{l}\text { Succinic } \\
\text { acid }\end{array}$ & Lactic acid & $\begin{array}{l}\text { Formic } \\
\text { acid }\end{array}$ & $\begin{array}{l}\text { Acetic } \\
\text { acid }\end{array}$ & $\begin{array}{l}\text { Propionic } \\
\text { acid }\end{array}$ & 2,3-Butanedio & Ethanol & $\begin{array}{l}\text { Butyric } \\
\text { acid }\end{array}$ & $\mathrm{pH}$ \\
\hline \multicolumn{10}{|c|}{ HMF } \\
\hline A & $0.62 \pm 0.31$ & $2.46 \pm 1.72$ & $0.00 \pm 0.00$ & $22.85 \pm 0.64$ & $0.63 \pm 0.17$ & $1.19 \pm 0.03$ & $1.88 \pm 0.01$ & $14.73 \pm 0.34$ & $6.32 \pm 0.02$ \\
\hline B & $0.43 \pm 0.61$ & $4.48 \pm 0.54$ & $0.00 \pm 0.00$ & $23.08 \pm 1.40$ & $0.87 \pm 0.05$ & $1.34 \pm 0.14$ & $1.79 \pm 0.39$ & $15.07 \pm 0.02$ & $6.08 \pm 0.11$ \\
\hline $\mathrm{C}$ & $1.47 \pm 0.65$ & $6.55 \pm 3.72$ & $0.00 \pm 0.00$ & $24.65 \pm 3.67$ & $0.66 \pm 0.24$ & $1.04 \pm 0.45$ & $1.47 \pm 0.17$ & $14.21 \pm 0.94$ & $6.00 \pm 0.14$ \\
\hline D & $2.14 \pm 0.67$ & $3.81 \pm 5.38$ & $4.48 \pm 6.33$ & $46.19 \pm 9.22$ & $2.66 \pm 0.11$ & $3.05 \pm 2.12$ & $3.06 \pm 2.19$ & $13.58 \pm 1.16$ & $5.65 \pm 0.07$ \\
\hline E & $0.00 \pm 0.00$ & $26.74 \pm 4.19$ & $0.00 \pm 0.00$ & $19.85 \pm 1.32$ & $1.24 \pm 0.07$ & $0.00 \pm 0.00$ & $1.24 \pm 1.03$ & $0.31 \pm 0.43$ & $6.06 \pm 0.08$ \\
\hline \multicolumn{10}{|c|}{ FUR } \\
\hline A & $1.84 \pm 0.28$ & $8.66 \pm 0.16$ & $0.00 \pm 0.00$ & $24.08 \pm 1.24$ & $1.15 \pm 0.26$ & $2.09 \pm 1.03$ & $2.38 \pm 0.74$ & $16.29 \pm 1.26$ & $6.25 \pm 0.07$ \\
\hline B & $0.81 \pm 1.14$ & $0.00 \pm 0.00$ & $0.00 \pm 0.00$ & $19.43 \pm 2.99$ & $1.41 \pm 0.50$ & $0.00 \pm 0.00$ & $1.40 \pm 0.51$ & $12.97 \pm 1.71$ & $5.88 \pm 0.06$ \\
\hline $\mathrm{C}$ & $5.65 \pm 2.17$ & $0.00 \pm 0.00$ & $0.00 \pm 0.00$ & $23.25 \pm 3.02$ & $1.78 \pm 0.02$ & $1.09 \pm 0.23$ & $2.30 \pm 0.85$ & $15.84 \pm 0.91$ & $5.66 \pm 0.08$ \\
\hline D & $1.13 \pm 0.03$ & $0.00 \pm 0.00$ & $0.00 \pm 0.00$ & $15.01 \pm 4.44$ & $1.38 \pm 0.72$ & $0.00 \pm 0.00$ & $0.00 \pm 0.00$ & $11.61 \pm 0.69$ & $5.75 \pm 0.10$ \\
\hline $\mathrm{E}$ & $5.25 \pm 7.43$ & $0.00 \pm 0.00$ & $0.00 \pm 0.00$ & $20.17 \pm 3.23$ & $1.99 \pm 0.05$ & $0.00 \pm 0.00$ & $1.32 \pm 0.19$ & $0.00 \pm 0.00$ & $5.39 \pm 0.02$ \\
\hline \multicolumn{10}{|c|}{$H M F+F U R$} \\
\hline A & $1.57 \pm 0.30$ & $8.34 \pm 1.31$ & $1.27 \pm 1.79$ & $25.61 \pm 3.72$ & $0.97 \pm 0.03$ & $3.69 \pm 2.15$ & $2.26 \pm 0.55$ & $16.50 \pm 0.36$ & $6.36 \pm 0.06$ \\
\hline B & $3.10 \pm 1.65$ & $0.00 \pm 0.00$ & $0.00 \pm 0.00$ & $14.13 \pm 0.68$ & $0.48 \pm 0.26$ & $0.00 \pm 0.00$ & $1.39 \pm 0.34$ & $11.92 \pm 1.40$ & $6.35 \pm 0.19$ \\
\hline $\mathrm{C}$ & $6.56 \pm 0.53$ & $0.00 \pm 0.00$ & $0.00 \pm 0.00$ & $28.06 \pm 0.15$ & $1.15 \pm 0.02$ & $6.80 \pm 0.36$ & $3.22 \pm 0.48$ & $16.21 \pm 0.27$ & $6.14 \pm 0.18$ \\
\hline D & $5.45 \pm 7.83$ & $0.00 \pm 0.00$ & $10.33 \pm 0.31$ & $47.34 \pm 5.14$ & $2.62 \pm 0.34$ & $15.38 \pm 2.09$ & $3.03 \pm 4.23$ & $15.58 \pm 0.28$ & $5.72 \pm 0.01$ \\
\hline E & $0.00 \pm 0.00$ & $30.68 \pm 2.14$ & $0.00 \pm 0.00$ & $19.84 \pm 0.21$ & $1.13 \pm 0.11$ & $7.73 \pm 0.51$ & $2.16 \pm 0.11$ & $0.00 \pm 0.00$ & $5.89 \pm 0.18$ \\
\hline
\end{tabular}


Table 3 Diversity analysis of the microbial community during the hydrogen fermentation with the addition of furan derivatives.

\begin{tabular}{lllllllll}
\hline \multirow{2}{*}{ Label } & Ace & \multicolumn{3}{c}{ Chao } & \multicolumn{3}{c}{ Shannon } & \multicolumn{2}{c}{ Simpson } \\
\cline { 2 - 9 } & HMF & FUR & HMF & FUR & HMF & FUR & HMF & FUR \\
\hline unique & 25624 & 24182 & 13871 & 13188 & 6.2 & 6.3 & 0.01 & 0.01 \\
$3 \%$ & 2934 & 2601 & 2529 & 2604 & 5.5 & 5.74 & 0.01 & 0.01 \\
$5 \%$ & 1988 & 1784 & 1733 & 1816 & 5.1 & 5.35 & 0.02 & 0.02 \\
$10 \%$ & 1003 & 1032 & 1013 & 1005 & 4.51 & 4.72 & 0.03 & 0.03 \\
\hline
\end{tabular}


Table 4 Comparison of dominant populations affected by HMF and FUR in genus level.

\begin{tabular}{llllll}
\hline Factor & $\begin{array}{c}\text { Anaerolineaceae } \\
\text { _uncultured }\end{array}$ & Clostridium & Leptolinea & Lactobacillus & $\begin{array}{c}\text { Ruminococcaceae } \\
\text { Incertae_Sedis }\end{array}$ \\
\hline HMF & 13.28 & 11.77 & 4.8 & 5.75 & 6.85 \\
FUR & 18.91 & 3.94 & 6.56 & 0.02 & 1.62 \\
\hline
\end{tabular}




\section{Figure captions}

Fig.1 Effect of additions of furan derivatives on hydrogen production from wet steam-exploded cornstalk in a 0.25 -L flask culture and changes of their concentrations $(\mathrm{n}=3)$. HMF, a and b; FUR, $\mathrm{c}$ and $\mathrm{d}$; HMF+FUR, e and $\mathrm{f}$. The concentrations of furan derivatives were 0 (A), 100 (B), 500 (C), 1000 (D) and $2000 \mathrm{mg} / \mathrm{L}$ (E).

Fig.2 Simultaneous degradation of furan derivatives and biohydrogen production from steam-exploded cornstalk with $8 \%$ TS in a 5-L stirred batch reactor culture $(n=3)$.

Fig.3 Rarefaction curve of microbial communities formed in hydrogen fermentation with additions of HMF or FUR based on relevant distances.

Fig.4 Taxonomic classification of pyrosequencing for the microbial communities formed in the fermentation with the additions of furan derivatives at the levels of phylum (A), class (B), order (C), family (D), genus (E), and species (F). 
Fig.1
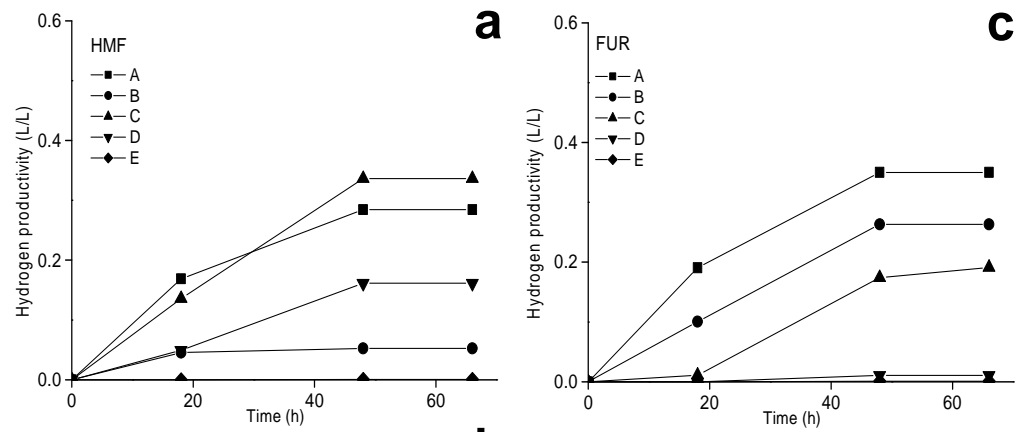

C ${ }^{0.6} 7_{\text {HMFFFUR }}$ e
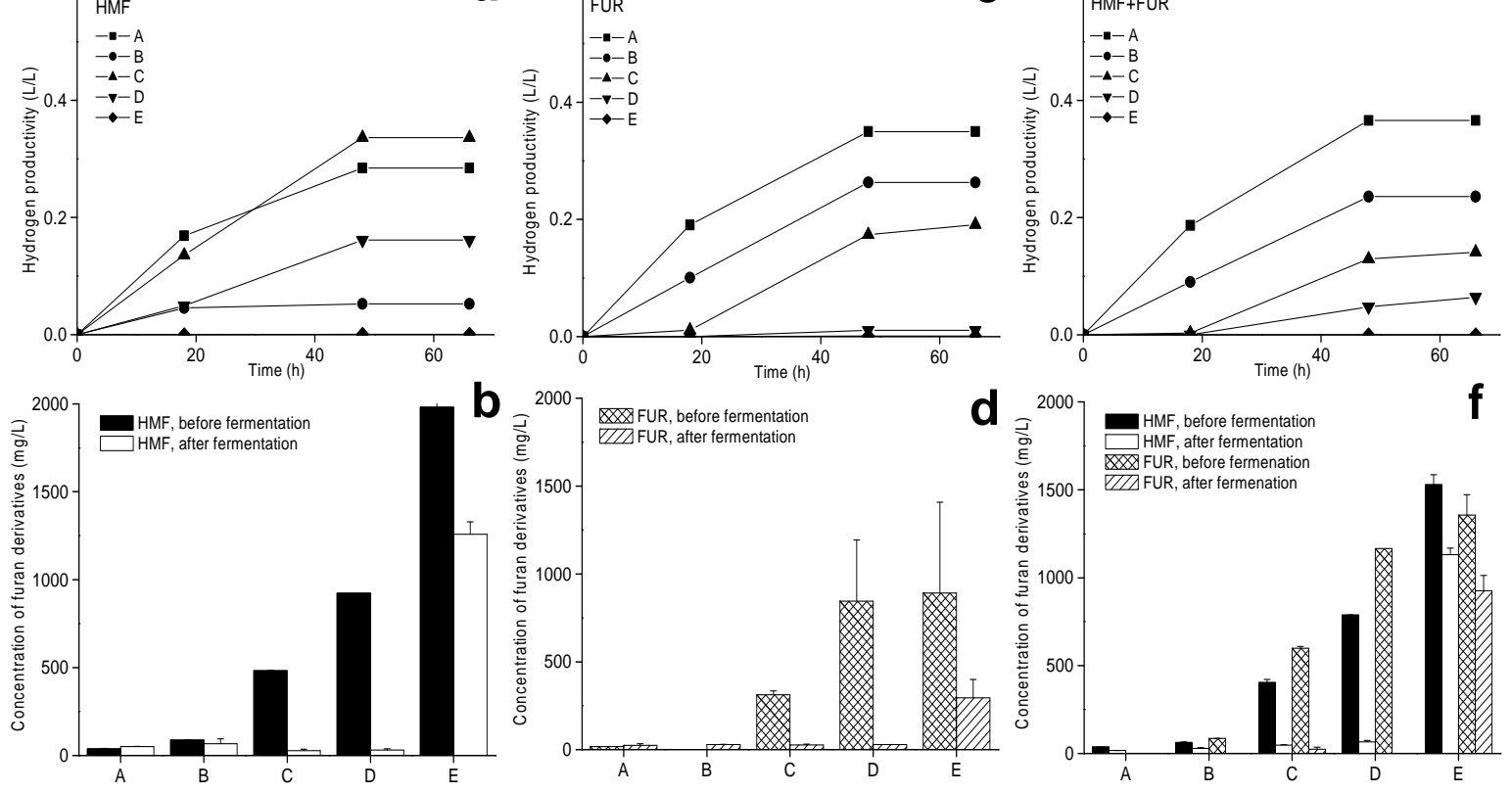
Fig.2

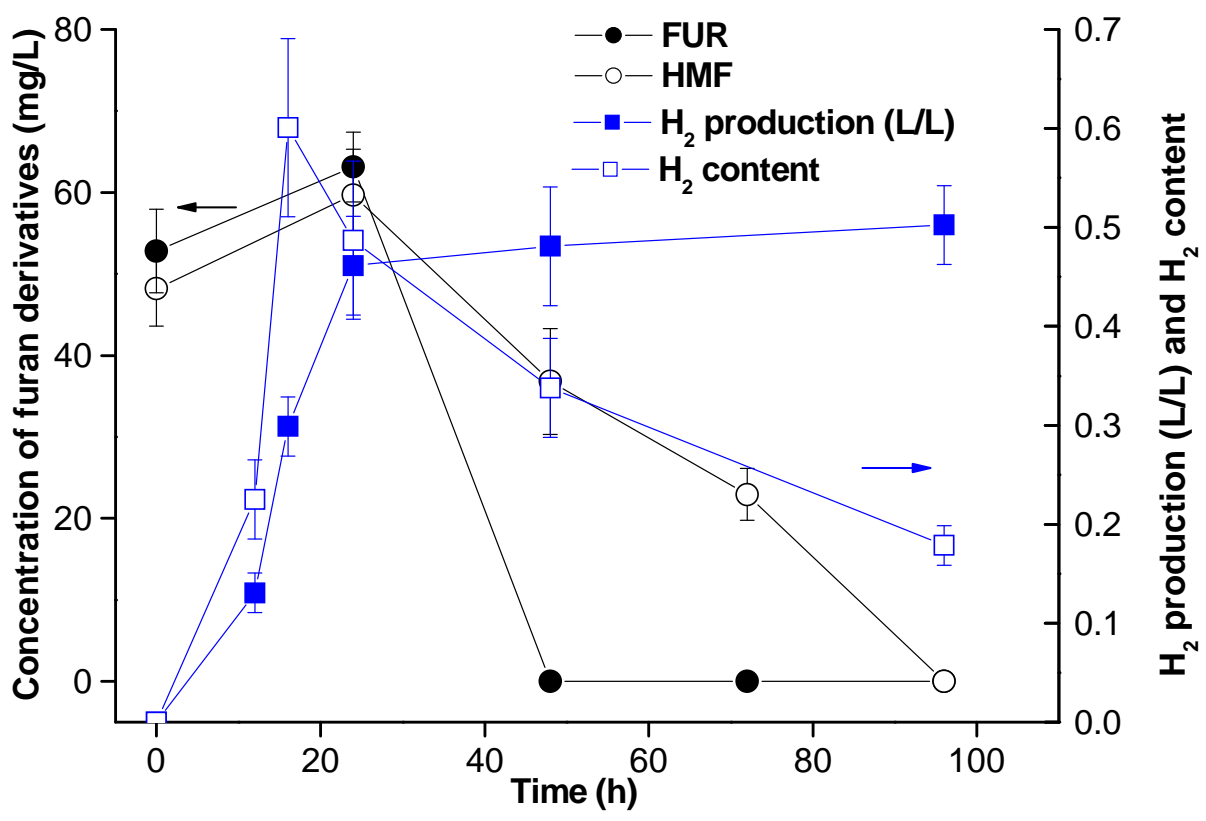


Fig.3

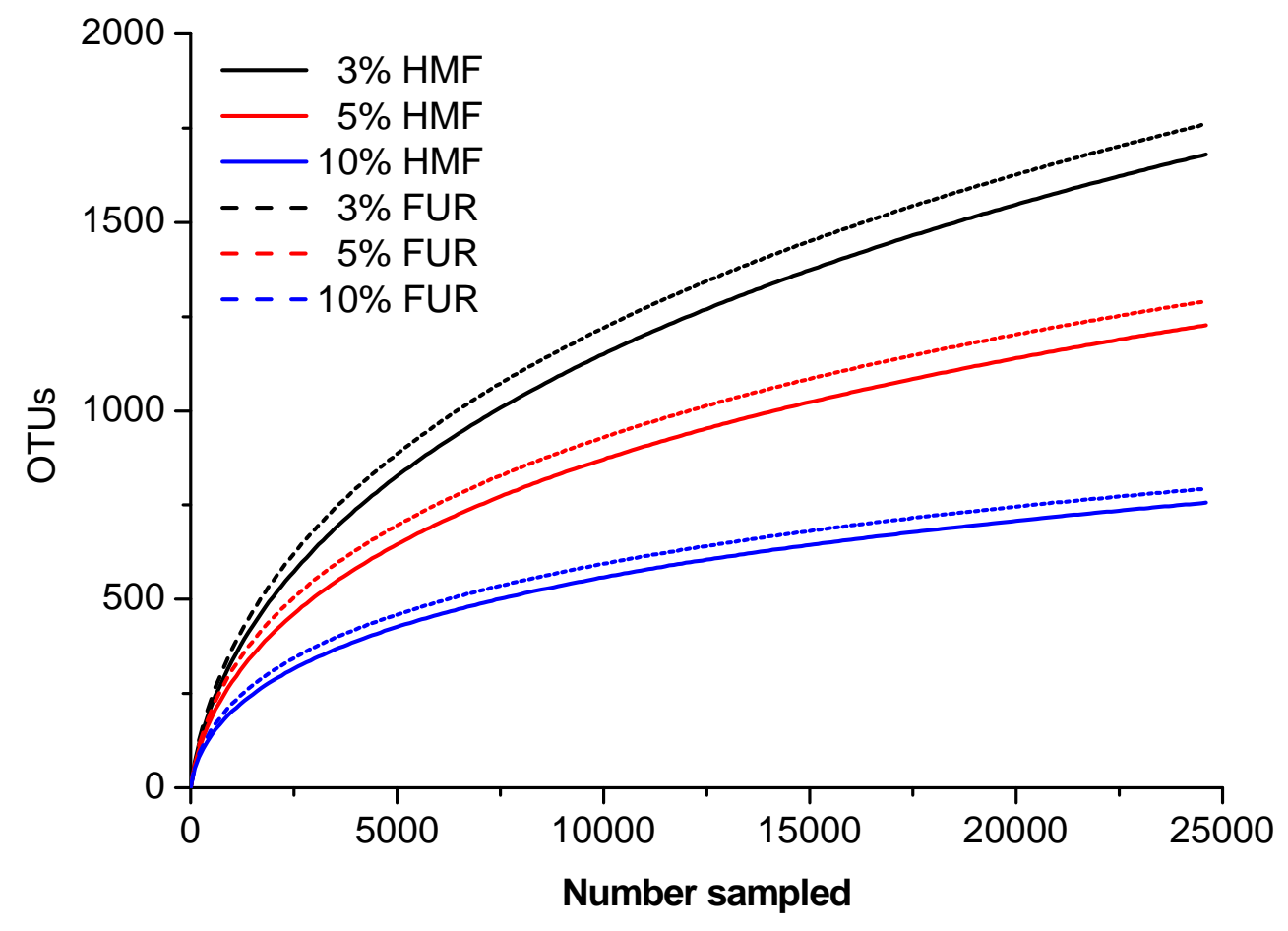


Fig.4
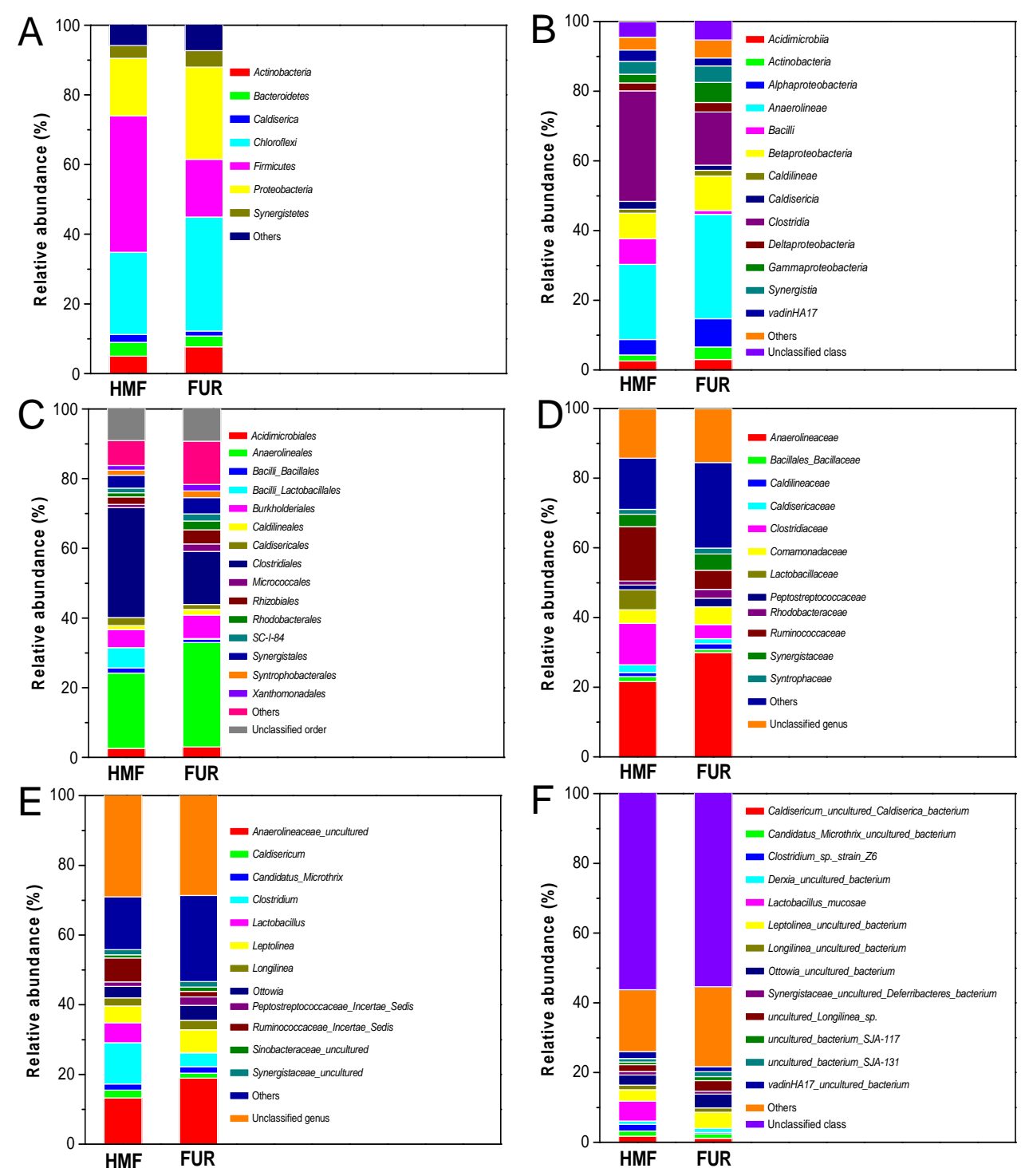